\title{
Preface
}

Fetal Diagnosis

and Therapy

Published online: February 26, 2015

\section{Prenatal Diagnosis and Treatment of Spina Bifida}

It has been a privilege to serve as the Guest Editor for this special issue of Fetal Diagnosis and Therapy about the prenatal diagnosis and treatment of spina bifida, with a focus on the most severe type of spina bifida, i.e. myelomeningocele (MMC). The contributors to this issue are mostly members of the Center for Fetal Diagnosis and Treatment at the Children's Hospital of Philadelphia (CHOP). Thus, the presentations reflect the philosophy of one center, gleaned from two decades of clinical and experimental work.

Studies in animal models and clinical case series laid the groundwork for a clinical trial to test the safety and efficacy of fetal MMC repair. The National Institute of Health-sponsored prospective, randomized Management of Myelomeningocele Study (the MOMS trial) demonstrated that fetal surgery for MMC before 26 weeks' gestation may preserve neurologic function, reverse hindbrain herniation, and obviate the need for postnatal placement of a ventriculoperitoneal shunt, but sig- nificant risks relate to the uterine scar and premature birth.

In this issue of Fetal Diagnosis and Therapy, the authors from CHOP along with colleagues from Belgium and New York City share findings and best practices gleaned from - and since - the MOMS trial, including the specific role different diagnostic and imaging modalities (maternal serum a-fetoprotein, ultrasound, MRI, and echocardiography) play in diagnosis, treatment and monitoring; nuances of the fetal MMC repair technique; longterm urologic functional outcomes; progress towards fetal MMC repair using tissue engineering techniques, and the fetal MMC repair outcomes at CHOP since the conclusion of the MOMS trial. Future research will evaluate the long-term impact of in utero intervention for spina bifida and refine the timing and technique of fetal MMC surgery.

N. Scott Adzick, MD, Philadelphia, Pa. Guest Editor 